\title{
Algorithm for parametric optimization of a trapezoidal wooden truss on metal tooth plates
}

\author{
Artur Zhurtov*, Tolya Hezhev, Artur Kardangushev, Muslim Nastuyev, Ismail Osmanov \\ Kabardino-Balkarian State University, 360004, Nalchik, Russia
}

\begin{abstract}
The article presents the research results on of a trapezoidal wooden truss with ascending braces on metal tooth plates parametric optimization. A deterministic algorithm has been developed and a program has been compiled that allows you to find the optimal parameters of the truss from the condition of minimizing the volume of wood, for given initial conditions. The optimal parameters for a truss with a span of 24 meters at a given value of the external load are revealed, diagrams of the dependence of the objective function on the varied parameters are drawn.
\end{abstract}

\section{Introduction}

In the world practice of construction production, a significant share is occupied by wooden structures. Over the past decades, constructive solutions and methods of joining elements of wooden structures have been actively improved. One of the modern and promising methods of joining wooden structures - metal tooth plates (hereinafter MTP). This fact is primarily due to the high technological and economic performance of this compound [1 - 4]. At the same time, there is a need to minimize labor and resource costs in the production of wooden structures on MTP. The solution to such problems is reduced to the compilation of an algorithm for optimizing the design parameters, at which the volume of wood will be minimal [5 - 10].

The article considered the possibility of compiling a deterministic parametric optimization algorithm for a trapezoidal wooden truss with ascending braces on MTP span L.

\section{Formulation of the problem}

A trapezoidal timber truss with ascending braces on MTP span $L$, which perceives the useful linear load $q$ is set, it is required to compose a deterministic algorithm, optimizing the following truss parameters:
a) $h$ - support height,
b) $\alpha$-inclination angle of the upper belt,
c) $n$ - number of truss panels,
d) $b$ - truss thickness,

* Corresponding author: zhurtovartur@mail.ru 
from the condition of minimizing the volume of wood used in the structure.

The loading scheme and the main parameters of the truss are shown in Fig. 1.

9

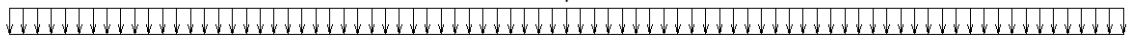

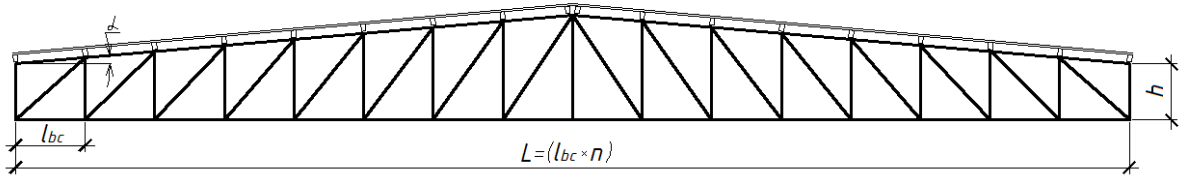

Fig. 1. Trapezoidal timber truss with upward braces on MTP span $L$

A method of connecting truss elements in nodes using MTP creates a number of restrictions for further optimization, the main of which is the same thickness for all elements. In addition, the joints flexibility on MTP is taken into account by checking the ultimate deformations, as required by the regulatory documents.

Unfastening from the truss plane is performed at each node of the upper chord.

\section{Algorithm for optimizing the truss parameters}

The proposed algorithm can be represented in the form of a schematic diagram shown in Fig. 2.

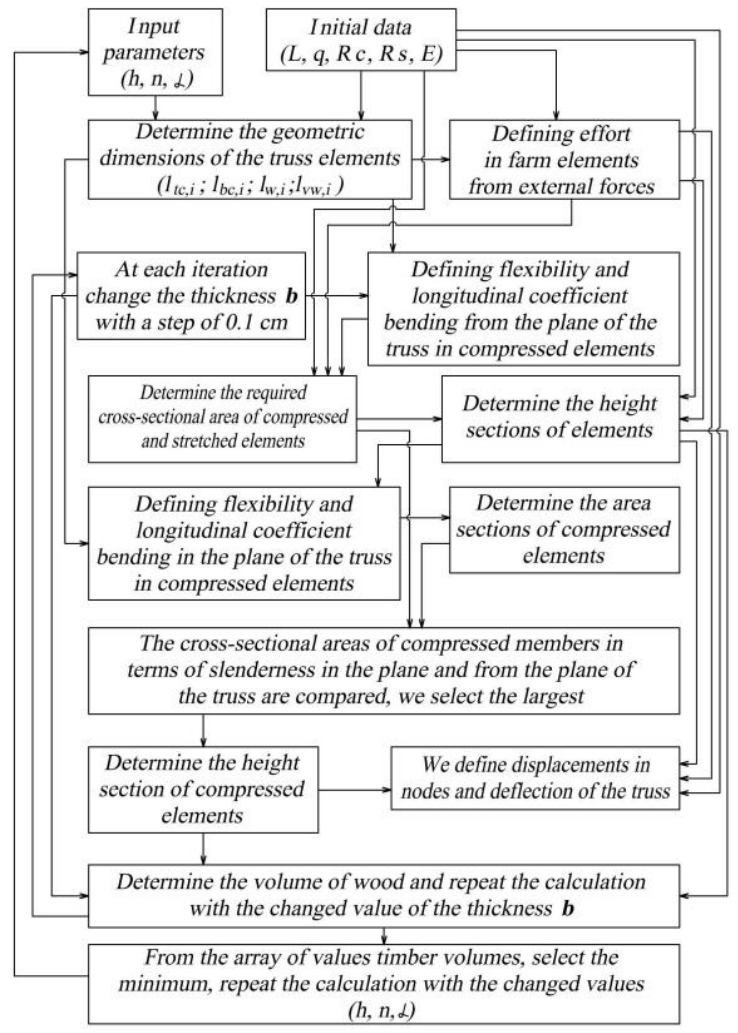

Fig. 2. Schematic algorithm diagram for finding the optimal parameters of the truss $h, n, \alpha, b$ by the wood minimum volume criterion 
The scheme for determining the geometric characteristics of the truss is shown in Fig. 3.

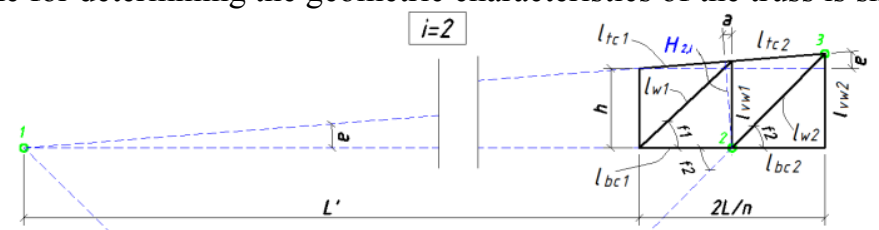

$H_{t, i}$

\section{$\mathfrak{9 0}^{90^{\circ}}$}

Fig. 3. Fragment of a trapezoidal truss, where $i$ is the number of the panel in question

From the diagram shown in Fig. 3 it follows that:

$$
\begin{gathered}
L^{\prime}=\frac{h}{\operatorname{tg} \alpha} ; \quad l_{t c}=\frac{L}{2 \cdot n \cdot \cos \alpha} ; \quad l_{b c}=\frac{L}{n} ; \quad l_{v w, i}=h+\frac{L \cdot i}{n} \cdot \operatorname{tg} \alpha ; \\
\varphi_{i}=\operatorname{arctg}\left(\frac{2 \cdot n \cdot\left(l_{v w, i}\right)}{L \cdot i}\right) ; \quad l_{w, i}=\sqrt{l_{b c}{ }^{2}+l_{v w}{ }^{2}} ; \\
H_{1, i}=\left(L^{\prime}+(i-1) \cdot l_{b c}\right) \cdot \sin \varphi_{i}=\left(\frac{h}{\operatorname{tg} \alpha}+(i-1) \cdot \frac{L}{n}\right) \cdot \sin \varphi_{i} \\
H_{2, i}=l_{v w,(i-1)} \cdot \cos \alpha=h+\frac{L \cdot(i-1)}{n} \cdot \sin \alpha
\end{gathered}
$$

The design scheme for determining the internal forces of the truss is shown in Fig. 4a and Fig. $4 \mathrm{~b}$.
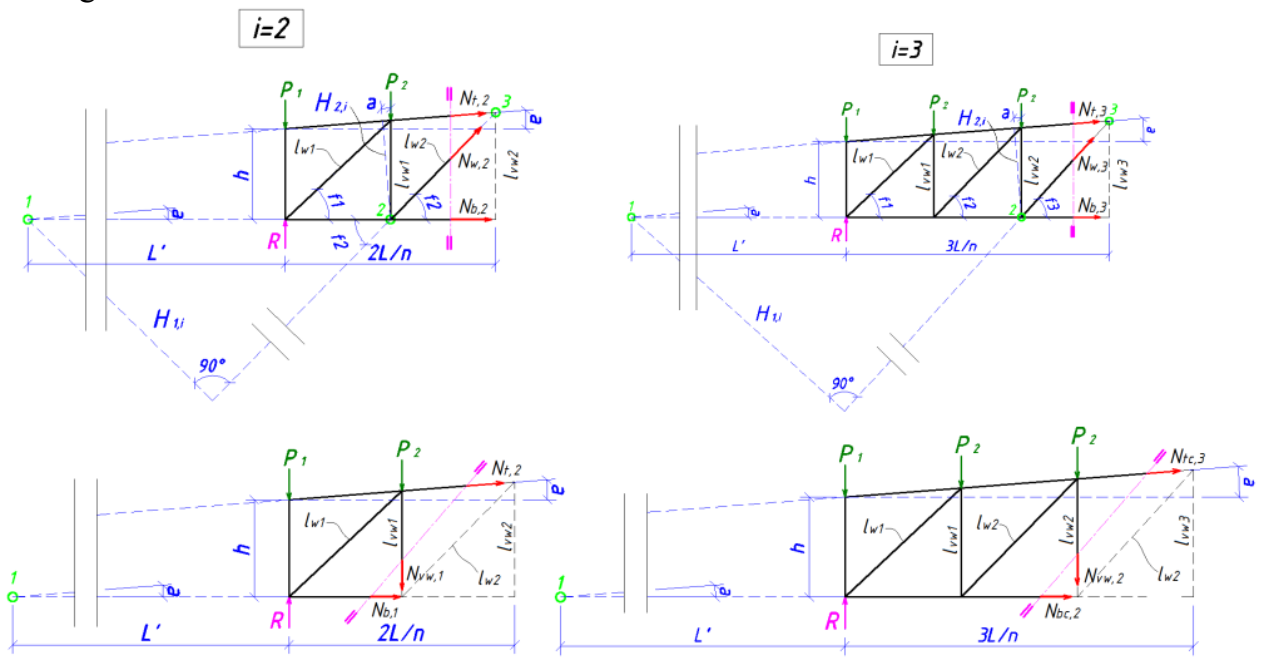

Fig. 4a and 4b. Scheme for determining the efforts in the 2nd and 3rd panels, respectively 
Let us express in general terms the internal efforts in the truss elements:

- in the $i$-th upper belt

$$
\begin{gathered}
N_{t, i}=\frac{\left(P_{1}-R\right) \cdot(i-1) \cdot l_{b c}}{H_{2, i}}+\frac{P_{2} \cdot l_{b c} \cdot\left(i^{2}-3 \cdot i+2\right)}{2 \cdot H_{2, i}}= \\
\frac{2 \cdot\left(P_{1}-R\right) \cdot(i-1) \cdot L+P_{2} \cdot L \cdot\left(i^{2}-3 \cdot i+2\right)}{2 \cdot H_{2, i} \cdot n}=\frac{2 \cdot\left(P_{1}-R\right) \cdot(i-1) \cdot L+P_{2} \cdot L \cdot\left(i^{2}-3 \cdot i+2\right)}{2 \cdot(h \cdot n+L \cdot(i-1) \cdot \sin \alpha)}
\end{gathered}
$$

- in the $i$-th rack

$$
N_{v w, i}=\frac{\left(R-P_{1}\right) \cdot \frac{h}{\operatorname{tg} \alpha}-P_{2} \cdot\left(\frac{i \cdot h}{\operatorname{tg} \alpha}+\frac{\left(i^{2}+i\right) \cdot L}{2 \cdot n}\right)}{\left(\frac{h}{\operatorname{tg} \alpha}+i \cdot \frac{L}{n}\right)}=\frac{2 \cdot n \cdot\left(R-P_{1}\right) \cdot h-P_{2} \cdot\left(2 \cdot n \cdot i \cdot h+\left(i^{2}+i\right) \cdot L \cdot \operatorname{tg} \alpha\right)}{2 \cdot(n \cdot h+i \cdot L \cdot \operatorname{tg} \alpha)} ;
$$

- in the $i$-th inclined brace

$$
N_{w, i}=\frac{\left(P_{1}-R\right) \cdot L^{\prime}+P_{2} \cdot\left((i-1) \cdot L^{\prime}+\left(\frac{i^{2}-i}{2}\right) \cdot l_{b c}\right)}{\left(L^{\prime}+(i-1) \cdot l_{b c}\right) \cdot \sin \varphi_{i}}
$$

- in the $i$-th lower belt

where

$$
N_{b c, i}=\frac{\left[i \cdot\left(R-P_{1}\right)-\frac{\left(i^{2}-i\right)}{2} \cdot P_{2}\right] \cdot l_{b c}}{l_{w, i}}=\frac{\left(i \cdot n-i^{2}\right) \cdot L \cdot P_{2}}{2 \cdot(h \cdot n+L \cdot i \cdot \operatorname{tg} \alpha)},
$$

$$
P_{1}=\frac{q \cdot L}{2 \cdot n} ; \quad P_{2}=2 \cdot P_{1}=\frac{q \cdot L}{n} ; R=\frac{q \cdot L}{2}
$$

In accordance with the algorithm shown in Fig. 2 we will define flexibility $\lambda_{y}$ and the buckling factor $\varphi_{y}$ from the plane of the truss for compressed members by the formulas

$$
\begin{gathered}
\lambda_{y}=\frac{l_{0}}{r_{y}} ; \quad r_{y}=0,289 \cdot b ; \\
\varphi_{y}=\frac{3000}{\lambda_{y}}, \text { if } \lambda_{y}>70 ; \\
\varphi_{y}=1-0,8 \cdot\left(\frac{100}{\lambda_{y}}\right)^{2}, \text { if } \lambda_{y} \leq 70 .
\end{gathered}
$$

where

$l_{0}$ defines element calculated length, $b$ is the truss width adjustable in $0.1 \mathrm{~cm}$ increments.

The required cross-sectional area and the height of the truss elements are determined by the well-known formula: 


$$
F_{r e q, y}=\frac{N}{\varphi_{y} \cdot R_{c} \cdot \gamma} ; \quad F_{r e q, y}=\frac{N}{R_{s} \cdot \gamma} ; h_{r e q, y}=\frac{F_{r e q, y}}{b}
$$

According to the section height value of the elements found above $h_{r e q, y}$ we will define their flexibility $\lambda_{x}$ and the buckling factor $\varphi_{x}$ in the plane of the truss.

$$
\begin{gathered}
\lambda_{x}=\frac{l_{0}}{r_{x}} ; \quad r_{x}=0,289 \cdot h_{r e q, y} ; \\
\varphi_{x}=\frac{3000}{\lambda_{x}}, \text { if } \lambda_{x}>70 ; \\
\varphi_{x}=1-0,8 \cdot\left(\frac{100}{\lambda_{x}}\right)^{2}, \text { if } \lambda_{x} \leq 70 .
\end{gathered}
$$

Next, we find the required cross-sectional area and the height of the compressed elements in terms of flexibility $\lambda_{x}$ in the plane of the truss:

$$
F_{r e q, x}=\frac{N}{\varphi_{x} \cdot R_{c} \cdot \gamma} ; \quad h_{r e q, x}=\frac{F_{r e q, x}}{b} .
$$

Let us compare $h_{\text {req,y }}$ and $h_{\text {req,x }}$ and choose the largest value. For all elements of the upper belt, we take the same height - the largest of $h_{\text {req }}$, in the same way for the lower belt.

The volume of wood used in the truss is determined by the formula

$$
V=2 \cdot l_{t b} \cdot b \cdot h_{t b} \cdot n+2 \cdot b \cdot \sum_{i=1}^{k}\left(h_{w, i} \cdot l_{w, i}\right)+2 \cdot b \cdot \sum_{i=1}^{k}\left(h_{v w, i} \cdot l_{v w, i}\right)+2 \cdot l_{b c} \cdot b \cdot h_{b c} \cdot n
$$

where

$$
k=n / 2 \text {. }
$$

The deflection of the truss is determined by Mohr's method, we load it with a vertical unit force applied in the middle of the lower chord. Let us determine the internal forces in the elements from a unit force:

$$
\begin{gathered}
\bar{N}_{t c, i}=\frac{(i-1) \cdot L}{2 \cdot(h \cdot n+L \cdot(i-1) \cdot \sin \alpha)} \\
\bar{N}_{v w, i}=\frac{n \cdot h}{2 \cdot(n \cdot h+i \cdot L \cdot \operatorname{tg} \alpha)} \\
\bar{N}_{w, i}=\frac{1}{2}+\frac{n \cdot h}{2 \cdot(i-1) \cdot L \cdot \sin \varphi_{i} \cdot \operatorname{tg} \alpha} \\
\bar{N}_{b c, i}=\frac{i \cdot L}{2 \cdot n \cdot h+2 \cdot L \cdot i \cdot \operatorname{tg} \alpha}
\end{gathered}
$$


then the deflection of the truss is:

$$
f=2 \cdot \sum_{i=1}^{k} \frac{N_{t c, i} \cdot \bar{N}_{t c, i}}{E F_{r e q, i}}+2 \cdot \sum_{i=1}^{k} \frac{N_{v w, i} \cdot \bar{N}_{v w, i}}{E F_{r e q, i}}+2 \cdot \sum_{i=1}^{k} \frac{N_{w, i} \cdot \bar{N}_{w, i}}{E F_{r e q, i}}+2 \cdot \sum_{i=1}^{k} \frac{N_{b c, i} \cdot \bar{N}_{b c, i}}{E F_{r e q, i}}
$$

If the truss deflection exceeds the permissible value, then, in accordance with the proposed algorithm, the thickness of the truss should be increased by $0.1 \mathrm{~cm}$ and recalculated. As a result of the calculation, an array of values $\backslash u 200 b \backslash u 200$ bof timber per truss is formed, depending on the width of the truss.

According to the proposed algorithm, a program was compiled in which it is possible, by changing the input parameters $L, q, h, n, \alpha$ both manually and automatically, optimize the parameters of the truss according to the criterion of the minimum volume of wood.

As an example, a trapezoidal wooden truss with ascending braces on MTP span $L=24 \mathrm{~m}$ loaded with payload $q=6 \mathrm{kN} / \mathrm{m}$, material grade I pine. It is required to determine such parameters, as the height on the support, the inclination angle of the upper chord, the number of truss panels, at which the consumption of wood would be minimal.

As a result of the calculation, the program generates data arrays, according to which the diagrams are shown in Fig. 5a and 5b. All volume values shown in Fig. 5a, except for those related to the height on the support less than $h=1,3 \mathrm{~m}$ inclusive, and in Fig. 5b, except for those related to the height on the support less than $h=1,1 \mathrm{~m}$ inclusive, satisfy the deformability conditions.
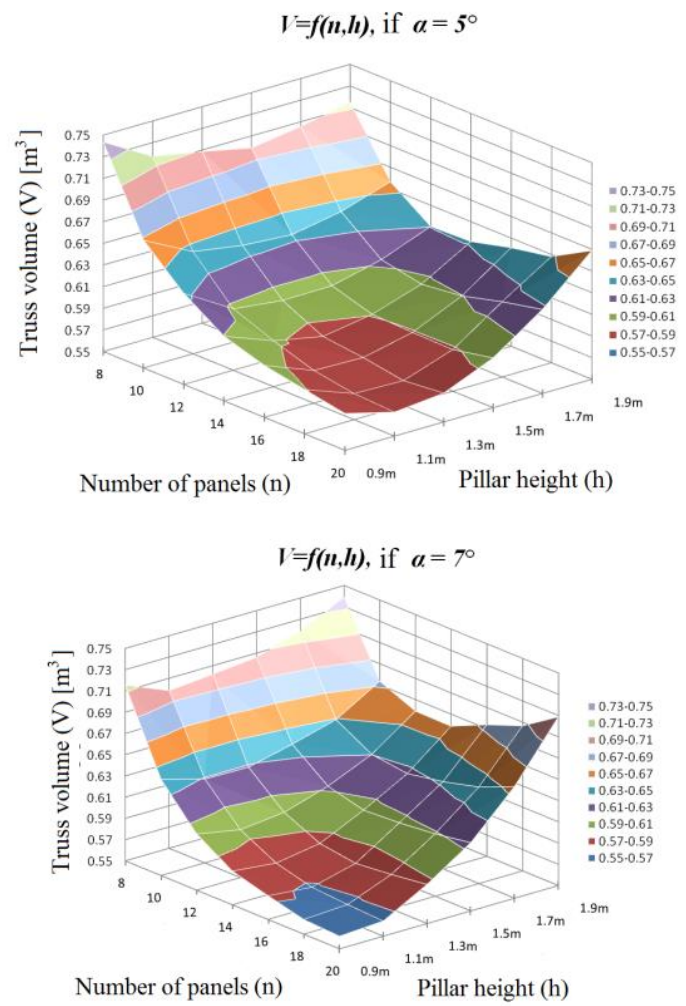

Fig. 5b. Wood volume objective function diagram $V$ from variable parameters $n$ and $h$, at $\alpha=7^{\circ}$ 
Analysis of the diagrams shows that for the original truss, the optimal parameters for the criterion of minimum wood consumption are $n=16, h=1,3 \mathrm{~m}, \alpha=7^{\circ}$.

\section{Conclusion}

The proposed deterministic algorithm and the program created on its basis make it possible to find the optimal parameters of a wooden trapezoidal truss with ascending braces on MTP from the condition of minimizing the volume of wood, given the initial conditions. As an example, a truss is optimized with a span of 24 meters with a given load of $6 \mathrm{kN} / \mathrm{m}$. This algorithm is applicable to trusses of various shapes, with minor changes to it regarding the geometric dimensions' determination of the lattice and the finding of internal forces in the truss elements.

\section{References}

1. V.G. Mironov, Privolzhsky scientific journal 4 (36), 45-54 (2015).

2. V.G. Kotlov, S.L. Mashinova, Industrial and civil construction 3, 53-54 (2003).

3. S.V. Fedosov, V.G. Kotlov, A.A. Aktuganov, Construction 11-12, 39-43 (2013).

4. T.L. Dmitriyeva, International Journal of Civil and Building Structures Calculation 9 (4), 128-136 (2013).

5. E.A. Sychev, A.A. Chinnov, Science Alley 1 (14), 425-429 (2017).

6. A.V. Kluyev, XVII Scientific Readings, International scientific and practical conference 3, 52-57 (2005).

7. A.P. Denisova, S.A. Rashchepkina, Methods for the optimal design of building structures (Publishing House of the Association of Construction Universities (ASV), 2012).

8. A.A. Vasilkin, E.K. Rakhmonov, Engineering Bulletin of Don 4 (2013). Information on ivdon.ru/ru/magazine/archive/n4y2013/2203.

9. E.V. Litvinova, International research journal 11 (53) Part 4 (2011).

10. R. Sacks, A. Warszawski, U. Kirsch, Automation in Construction 10 (1), 181-197 (2000). 